\author{
向光素 LOV1-C57S 固态 Photo-CIDNP 效应的磁场依赖性 \\ 王孝杰*,a Smitha Surendran Thamarath ${ }^{b, c} \quad J o ̈ r g$ Matysik(誉宫)*,b,c \\ ( ${ }^{a}$ 国防科技大学理学院化学与生物学系 长沙 410073$)$

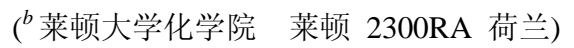 \\ ( 莱比锡大学分析化学系 莱比锡 04103 德国)
}

\begin{abstract}
摘要 利用 ${ }^{13} \mathrm{C}$ 魔角旋转 NMR 波谱对向光素 LOV1-C57S 进行固态 Photo-CIDNP 效应的磁场依赖性研究. 在 $4.7 \mathrm{~T}$ (相 当于 $200 \mathrm{MHz}{ }^{1} \mathrm{H}$ 频率)磁场中, 黑暗条件下只检测到较宽的蛋白质信号; 但在光照条件下, 观测到强烈的固态 Photo-CIDNP 效应. 与在 $2.3 \mathrm{~T}$ (相当于 $100 \mathrm{MHz}{ }^{1} \mathrm{H}$ 频率)磁场中得到的全部为发射谱(负)信号的固态 Photo-CIDNP 图谱 (首次在非光合反应系统中观测到的 Photo-CIDNP 效应)不同, 在 $4.7 \mathrm{~T}$ 磁场中测得的光诱导 ${ }^{13} \mathrm{C} \mathrm{NMR}$ 谱呈现吸收/发射 混合峰型, 这种峰型与文献报道的 LOV2 的液态Photo-CIDNP 波谱的峰型相似. 对比两种磁场中所得谱图发现, 向光素 LOV1-C57S 的固态 Photo-CIDNP 效应具有强烈的磁场依赖性，而且不同的核对磁场的依赖程度差别很大，这种巨大的 差别反映出在被测试的自由基对中的超精细相互作用影响因素发生了很大的改变.

关键词 光化学诱导动态核极化; 魔角旋转; 核磁共振; 向光素; LOV 结构域
\end{abstract}

\title{
Magnetic Field Dependence of the Solid-State Photo-CIDNP Effect Ob- served in Phototropin LOV1-C57S
}

\author{
Wang, Xiaojie Smitha Surendran Thamarath $^{b, c} \quad$ Jörg Matysik*,b,c $^{*, a}$ \\ ( ${ }^{a}$ Department of Chemistry and Biology, College of Science, National University of Defense Technology, Changsha 410073) \\ ( ${ }^{b}$ Leiden Institute of Chemistry, Einsteinweg 55, Leiden, 2300RA The Netherlands) \\ ( ${ }^{c}$ Institut für Analytische Chemie, Universität Leipzig, Linnéstr 3, Leipzig, 04103 Germany)
}

\begin{abstract}
Here we present the magnetic field dependence of the solid-state Photo-CIDNP effect observed in phototropin LOV1-C57S using ${ }^{13} \mathrm{C}$ magic-angle spinning (MAS) NMR spectroscopy. Both dark and light spectra were measured at $4.7 \mathrm{~T}$ (i.e., $200 \mathrm{MHz}{ }^{1} \mathrm{H}$ frequency) using a spinning frequency of $8 \mathrm{kHz}$. An Avance $200 \mathrm{MHz}$ spectrometer equipped with 4-mm MAS probe (Bruker, Karlsruhe, Germany) was used for the ${ }^{13} \mathrm{C}$ MAS NMR experiments. The sample was packed into a 4-mm sapphire rotor and inserted into the MAS probe. For a homogeneous sample distribution against the rotor wall, the sample was frozen at a very low spinning frequency of $500 \mathrm{~Hz}$. Freezing was monitored on the proton tuning channel as a shift of ca. 0.1 MHz. The variable temperature unit on the spectrometer was set to $235 \mathrm{~K}$. The spinning frequency was increased to $8 \mathrm{kHz}$ after the sample is completely frozen. This frequency and set temperature were used for all ${ }^{13} \mathrm{C}$ MAS NMR measurements. A simple Hahn-echo pulse sequence with two-pulse phase modulation proton-decoupling was used. Continuous illumination was supplied by a $1 \mathrm{~kW}$ xenon lamp. The cycle delay was $2 \mathrm{~s}$, and the measurement time was about $12 \mathrm{~h}$. In the spectrum obtained in the dark, standard broad protein responses appear. Under illumination, several strong additional light-induced signals appeared. In contrast to the entirely emissive (negative) peaks in the photo-CIDNP MAS NMR spectra observed at $2.3 \mathrm{~T}$ (i.e., $100 \mathrm{MHz}{ }^{1} \mathrm{H}$ frequency), the first observation of this effect in a nonphotosynthetic system, the light induced ${ }^{13} \mathrm{C}$ NMR peaks at $4.7 \mathrm{~T}$ show mixed absorptive/emissive enhancement pattern. This pattern is reminiscent of the spectra observed by liquid state photo-CIDNP of a LOV2 sample. The observed solid-state photo-CIDNP effect is strongly magnetic field dependent, and this field-dependence is well distinguished for the various nuclei. This large difference in magnetic field dependence reflects the large variety of hyperfine factors found in this comparable small-sized and asymmetric radical pair.
\end{abstract}

Keywords photo-CIDNP; MAS; NMR; phototropin; LOV

\section{1 引言}

光化学诱导动态核极化(Photo-Chemically Induced
Dynamic Nuclear Polarization, Photo-CIDNP)是一种在光 激发下形成自旋相关的自由基对(Radical Pair), 初始形

*E-mail: yj605@126.com; joerg.matysik@uni-leipzig.de

Received December 23, 2012; published January 17, 2013.

Supporting information for this article is available free of charge via the Internet at http://sioc-journal.cn.

Project supported by the Netherlands Organization for Scientific Research (NOW) through a Middelgroot grant (ALW open competitie, 818.02.019; Middelgroot 700.57.107).

项目受荷兰科学研究组织(NOW) (ALW open competitie, 818.02.019; Middelgroot 700.57.107)资助. 
成的自由基对具有很高的电子自旋有序性，这种电子自 旋的有序性在一定条件下可以传递给核，从而使核自旋 极化产生明显偏离玻尔兹曼布居的效应. 这种效应使 NMR 波谱出现增强的吸收谱(正)或发射谱(负)信号, 因 此, 可以通过 NMR 波谱信号强度的变化来观测这种效 应. 由于 Photo-CIDNP 效应是在光化学反应过程中产生 的, 所以, 利用这种效应可以直接研究光诱导电子转移 过程的机理.

Photo-CIDNP 效应在液体 NMR 中的应用早在 1967 年 ${ }^{[1,2]}$ 就已经开始, 其相关理论也已经比较成熟. 然而, 由于液体 NMR 对样品分子量的限制, 使得其应用大多 局限于对小分子的研究. 对于像生命过程中的光化学反 应这类涉及蛋白质大分子的反应过程的研究, 通常很难 观测到液态 Photo-CIDNP 效应 ${ }^{[3]}$.

利用固体 NMR 可以解决样品分子量受限的问题. 但由于固态 Photo-CIDNP 效应受研究对象影响较大且 对检测条件要求较高, 直到 1994 年才第一次被观测到, 是 McDermott 小组 ${ }^{[4]}$ 在对类球红杆菌的光合反应中心进 行 ${ }^{13} \mathrm{C}$ 魔角旋转核磁共振(MAS NMR)测试时发现的. 实 验发现这种效应可使信号增强数万倍 ${ }^{[5,6]}$. 如此强烈的 信号增强使得实验可以直接以膜、细胞甚至整个组织为 样品, 其中仅有纳摩尔量级浓度的反应物信号都可以有 效地被观测到. 此后, 对各种天然光合反应中心开展了 固态 Photo-CIDNP 效应的研究 ${ }^{[7 \sim 9]}$, 相关理论也逐渐发 展起来 ${ }^{[10 ~ 14]}$. 但在很长一段时间里, 尽管人们做了大量 的努力, 在非光合反应系统中的实验都失败了 ${ }^{[15]}$.

最近, 我们在莱茵衣藻(一种绿藻)向光素的突变体 LOV1-C57S 中观测到了固态 Photo-CIDNP 效应 ${ }^{[16]}$, 这 是首次在非光合反应系统中发现这种效应. 这个实验是 在 $2.3 \mathrm{~T}$ (相当于 $100 \mathrm{MHz}{ }^{1} \mathrm{H}$ 频率)磁场中连续光照条件 下进行的, 它证明固态 photo-CIDNP 效应不只局限于天 然光合反应系统中, 它有可能发展成为一种普遍通用的 信号增强的方法.

向光素是一种蓝光受体, 其主要作用是调控植物向 光性运动、叶绿体移动及气孔开放等光响应过程 ${ }^{[17,18]}$. 向光素包含两个光-氧 - 电位 (Light-Oxygen-Voltage, LOV)敏感结构域(LOV1 和 LOV2), 两个 LOV 结构域各 自与一个黄素单核苷酸(FMN)非共价结合作为发色团. 在光照条件下, LOV1 的 FMN 与邻近的一个半胱氨酸残 基(Cys57)共价结合形成信号态复合物 ${ }^{[19]}$. 我们研究的 向光素突变体 LOV1-C57S 是将参加反应的 Cys57 突变 为丝氨酸, 从而阻断了上述复合物的形成. 在光照时, 由于 FMN 与 Cys57 形成复合物的优势路径被阻断，一 条原本在竞争中处于劣势的路径得以发生, 即电子从一 个色氨酸残基(Trp98)转移到 FMN, 形成由 FMN 和 Trp98 组成的自由基对 ${ }^{[20]}$. 这个原初自由基对的形成使 系统产生固态 Photo-CIDNP 效应.

我们对向光素 LOV1-C57S 固态 Photo-CIDNP 效应
的磁场依赖性进行了研究, 分别在一系列不同的磁场中 对其进行光照条件下的 ${ }^{13} \mathrm{C}$ MAS NMR 测试. 其中, 在 $4.7 \mathrm{~T}$ (相当于 $200 \mathrm{MHz}{ }^{1} \mathrm{H}$ 频率)磁场中, 也观测到了显 著的固态 Photo-CIDNP 效应, 但其谱图与在 $2.3 \mathrm{~T}$ 磁场 中所得谱图有较大区别, 本文报道了相关结果.

\section{2 结果与讨论}

\subsection{LOV1-C57S 在 4.7 T 磁场中的固态 Photo-CIDNP 效应}

在 LOV1-C57S 中观测到固态 Photo-CIDNP 效应之 后，我们进一步开展了在不同磁场强度条件下的实验. 图 1 为向光素 LOV1-C57S 在 $4.7 \mathrm{~T}$ 磁场中的 ${ }^{13} \mathrm{C} \mathrm{MAS}$ NMR 波谱. 测试条件参照文献[16]为: 磁场强度 $4.7 \mathrm{~T}$, 魔角旋转频率 $8 \mathrm{kHz}$, 回波延迟时间为 $125 \mathrm{~ms}$, 测试温 度 $235 \mathrm{~K}$. 整个 NMR 测试过程中, 样品始终处于冰冻状 态. 在双脉冲相位调制一质子去耦条件下采用简单的哈 恩回波脉冲序列. 连续光源为一个 $1 \mathrm{~kW}$ 的氙灯. 周期 延迟为 $2 \mathrm{~s}$, 测量时间为 $12 \mathrm{~h}$.

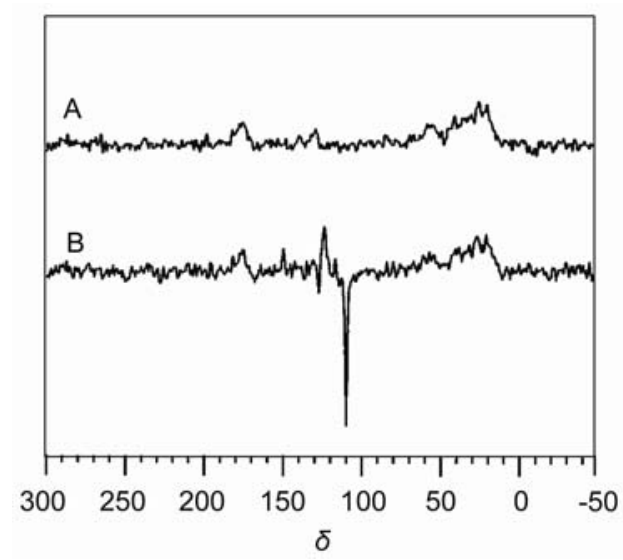

图 1 向光素 LOV1-C57S 在 $4.7 \mathrm{~T}$ 磁场中分别在黑暗条件下(A)及连 续白光照射条件下(B)的 ${ }^{13} \mathrm{C}$ MAS NMR 波谱

Figure $1{ }^{13} \mathrm{C}$ MAS NMR spectra of phototropin LOV1-C57S obtained at a magnetic field of $4.7 \mathrm{~T}$ in the dark (A) and under continuous illumination with white light (B)

从图 1 可以看出, 在黑暗条件下只检测到较宽的信 号, 这是标准的蛋白质信号, 但在光照条件下, 出现了 几条强烈的光诱导信号. 从图谱中光诱导信号化学位移 的位置来看，与在 $2.3 \mathrm{~T}$ 磁场中得到的固态 Photo-CIDNP 图谱(图 2)一致, 说明, 在 4.7 $\mathrm{T}$ 磁场中也 观测到固态 Photo-CIDNP 效应.

\subsection{LOV1-C57S 固态 Photo-CIDNP 效应磁场依赖性 分析}

固态 Photo-CIDNP MAS NMR 信号来自组成自由 基对的电子给体和电子受体. 分析信号的化学位移可以

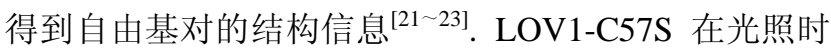
会形成以 FMN 为电子受体的自由基对. 图 2 给出了分 
别在 $2.3 \mathrm{~T}$ 和 $4.7 \mathrm{~T}$ 磁场中光照条件下获得的固态 Photo-CIDNP MAS NMR 谱图, 图中显示为光诱导信号 区域的放大图.

从图 2 可以看出, 在两个不同磁场中测得的固态 Photo-CIDNP 波谱主要信号的化学位移基本一致, 表明 在两个实验中形成的自由基对组成是相同的. 然而两条 谱线的形状却有较大差别, 在 $2.3 \mathrm{~T}$ 磁场中测得的波谱 全部为发射谱(负)信号, 在 $4.7 \mathrm{~T}$ 磁场中测得的光诱导 ${ }^{13} \mathrm{C}$ NMR 谱呈现吸收/发射混合峰型, 这种峰型与 Weber 小组 ${ }^{[20]}$ 在 $11.7 \mathrm{~T}$ 磁场中测得的 LOV2 的液态 PhotoCIDNP 波谱的峰型相似.

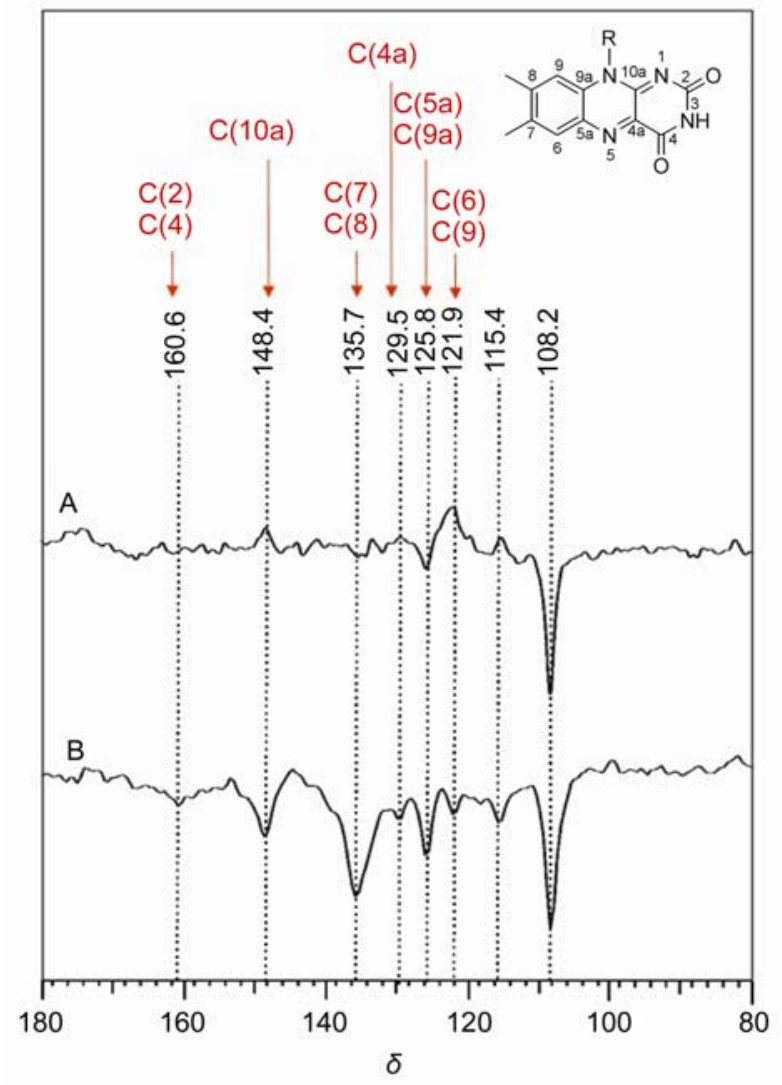

图 2 向光素 LOV1-C57S 分别在 4.7 T (A)和 $2.3 \mathrm{~T}$ (B)磁场中光照条 件下的固态 ${ }^{13} \mathrm{C}$ MAS NMR 波谱光诱导信号区域的放大图

Figure 2 Expanded view on the light-induced signal region of the ${ }^{13} \mathrm{C}$ MAS NMR spectra of phototropin LOV1-C57S under illumination at a magnetic field of $4.7 \mathrm{~T}(\mathrm{~A})$ and $2.3 \mathrm{~T}(\mathrm{~B})$

The flavin chromophore is shown at the top and the numbering of the isoalloxazine ring is included. The red labels indicate the signals assigned to the carbons in the isoalloxazine ring

对比在两种磁场中所得谱图发现, 在 $4.7 \mathrm{~T}$ 磁场中, $\mathrm{C}(5 \mathrm{a})$ 和 C(9a)的 NMR 发射信号基本保持原状, C(7)和 $C(8)$ 虽然仍保持发射信号, 但强度明显削弱, C(2)和 $\mathrm{C}(4)$ 信号基本消失, C(6), C(9), C(4a)和 C(10a)探测到的 是吸收信号. 信号峰型在不同磁场中发生明显变化说明 观测到的固态 Photo-CIDNP 效应具有强烈的磁场依赖 性. 各个碳核信号变化都不一样, 说明不同的核对磁场 的依赖程度差别很大. 目前的研究认为, 三旋混合机理
(Three-Spin Mixing, TSM) 是在连续光照实验中产生固 态 Photo-CIDNP 效应的主要机理之一[10,24], 这个机理与 超精细相互作用相关. 各个碳核信号变化的巨大差别反 映出在产生固态 Photo-CIDNP 效应的自由基对的相对 较小的区域内存在着复杂的超精细相互作用的影响因 素, 这些因素在不同的磁场环境中发生了很大的改变.

\section{3 结论}

利用 ${ }^{13} \mathrm{C}$ MAS NMR 波谱在 $4.7 \mathrm{~T}$ 磁场中观察到向 光素 LOV1-C57S 的固态 Photo-CIDNP 效应, 所得谱图 中主要信号的化学位移与在 $2.3 \mathrm{~T}$ 磁场中测得的固态 Photo-CIDNP 波谱基本一致，表明在两个实验中形成的 自由基对组成是相同的. 在两个不同磁场中所得谱图在 峰型上有较大区别, 说明自由基对产生固态 PhotoCIDNP 效应受磁场的影响, 而且, 不同的核受影响的程 度不同, 具有强烈的磁场依赖性.

致谢 感谢比勒费尔德大学的 Tilman Kottke 博士和 Elena Herma 为本工作提供向光素 LOV1-C57S 样品. 感 谢 B. Bode 博士(圣安德鲁斯大学)和 G. Jeschke 教授(苏 黎世联邦理工学院)在论文撰写过程中给予的积极讨论. 衷心感谢 F. Lefeber, K. Erkelens 和 K. B. Sai Sankar Gupta 博士在整个实验过程中给予的帮助.

\section{References}

[1] Bargon, J.; Fischer, F.; Johnson, U. Z. Naturforsch. A 1967, 22, 1551.

[2] Ward, H. R.; Lawler, R. G. J. Am. Chem. Soc. 1967, 89, 5518.

[3] Richter, G.; Weber, S.; Römisch, W.; Bacher, A.; Fischer, M.; Eisenreich, W. J. Am. Chem. Soc. 2005, 127, 17245.

[4] Zysmilich, M.; McDermott, A. J. Am. Chem. Soc. 1994, 116, 8362.

[5] Prakash, S.; Alia; Gast, P.; de Groot, H. J. M.; Jeschke, G.; Matysik, J. J. Am. Chem. Soc. 2005, 127, 14290.

[6] Prakash, S.; Alia; Gast, P.; de Groot, H. J. M.; Matysik, J.; Jeschke, G. J. Am. Chem. Soc. 2006, 128, 12794.

[7] Alia; Roy, E.; Gast, P.; van Gorkom, H. J.; de Groot, H. J. M.; Jeschke, G.; Matysik, J. J. Am. Chem. Soc. 2004, 126, 12819.

[8] Diller, A.; Roy, E.; Gast, P.; van Gorkom, H. J.; de Groot, H. J. M.; Glaubitz, C.; Jeschke, G.; Matysik, J.; Alia, A. Proc. Natl. Acad. Sci. U. S. A. 2007, 104, 12767.

[9] Janssen, G. J.; Daviso, E.; van Son, M.; de Groot, H. J. M.; Alia, A. Matysik, J. Photosynth. Res. 2010, 104, 275.

[10] Jeschke, G. J. Chem. Phys. 1997, 106, 10072.

[11] McDermott, A.; Zysmilich, M. G.; Polenova, T. Solid State Nucl. Magn. Reson. 1998, 11, 21.

[12] Polenova, T.; McDermott, A. E. J. Phys. Chem. B 1999, 103, 535.

[13] Jeschke, G.; Matysik, J. Chem. Phys. 2003, 294, 239.

[14] Daviso, E.; Jeschke, G.; Matysik, J. J. Phys. Chem. C 2009, 113, 10269.

[15] Matysik, J.; Diller, A.; Roy, E.; Alia, A. Photosynth. Res. 2009, 102, 427.

[16] Thamarath, S. S.; Heberle, J.; Hore, P.; Kottke, T.; Matysik, J. J. Am. Chem. Soc. 2010, 132, 15542.

[17] Christie, J. M.; Reymond, P.; Powell, G. K.; Bernasconi, P.; Raibekas, A. A.; Liscum, E.; Briggs, W. R. Science 1998, 282, 1698.

[18] Christie, J. M. Annu. Rev. Plant Biol. 2007, 58, 21.

[19] Salomon, M.; Christie, J. M.; Knieb, E.; Lempert, U.; Briggs, W. R. Biochemistry 2000, 39, 9401.

[20] Eisenreich, W.; Joshi, M.; Weber, S.; Bacher, A.; Fischer, M. J. Am. Chem. Soc. 2008, 130, 13544.

[21] Prakash, S.; Alia, A.; Gast, P.; de Groot, H. J. M.; Jeschke, G.; 
Matysik, J. Biochemistry 2007, 46, 8953.

[22] Roy, E.; Rohmer, T.; Gast, P.; Jeschke, G.; Alia, A.; Matysik, J. Biochemistry 2008, 47, 4629.

[23] Thamarath, S. S.; Bode, B. E.; Prakash, S.; Karthick, B. S. S. G.;
Alia, A.; Jeschke, G.; Matysik, J. J. Am. Chem. Soc. 2012, 134, 5921.

[24] Jeschke, G. J. Am. Chem. Soc. 1998, 120, 4425.

(Zhao, C.) 
Communication

Magnetic Field Dependence of the Solid-State Photo-CIDNP Effect Observed in Phototropin LOV1-C57S

Wang, Xiaojie*; Smitha Surendran Thamarath; Jörg Matysik*

Acta Chim. Sinica 2013, 71(X), XXX-XXX

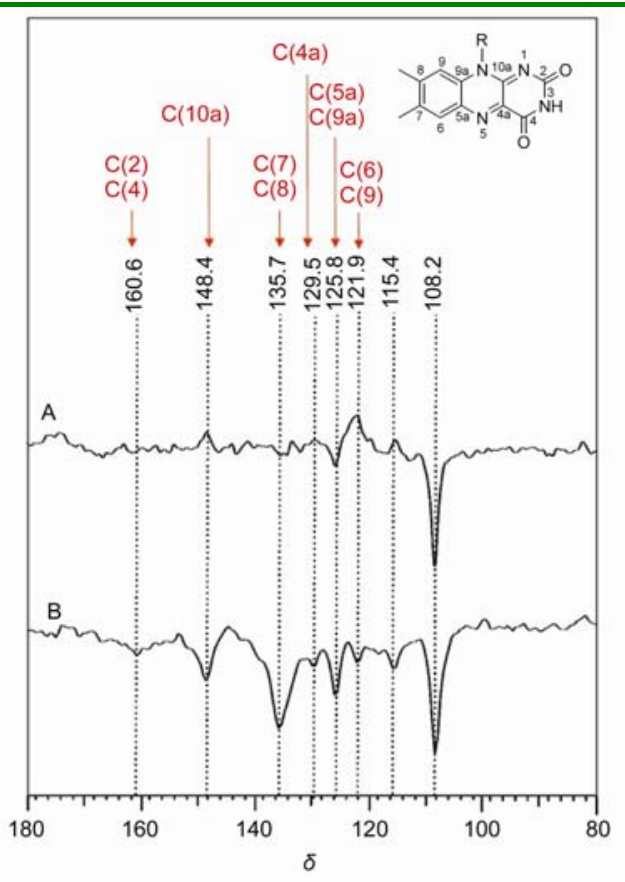

The magnetic field dependence of the solid-state Photo-CIDNP effect was observed in phototropin LOV1-C57S using ${ }^{13} \mathrm{C}$ MAS NMR Spectroscopy. The observed solid-state photo-CIDNP effect is strongly magnetic field dependent, and this field-dependence is very different for the various nuclei. 